EPiC Series in Engineering
Volume 3, 2018, Pages 1971-1978
HIC 2018. 13th International
Conference on Hydroinformatics

\title{
Complex Network Theory for Water Distribution Networks analysis
}

\author{
Antonietta Simone ${ }^{1 *}$, Luca Ridolfi ${ }^{2}$, Luigi Berardi ${ }^{1}$, Daniele B. Laucelli ${ }^{1}$ \\ and Orazio Giustolisi ${ }^{1}$ \\ ${ }^{1}$ Politecnico di Bari, Via E. Orabona 4, 70125 Bari, Italy \\ ${ }^{2}$ Politecnico di Torino, C.so Duca degli Abruzzi, 2410129, Torino, Italy \\ antonietta.simonelpoliba.it, luca.ridolfi@polito.it, \\ luigi.berardi@poliba.it, danielebiagio.laucelli@poliba.it, \\ orazio.giustolisiepoliba.it
}

\begin{abstract}
Performance of networked systems greatly depends on their topologic or connectivity structure. Nowadays, the analysis of the relevant features influencing the emerging behavior of networked systems is possible because of the increasing computational power and availability of information. Complex Network Theory classifies the connectivity structures of real systems using the nodal degree, the average path length, the clustering coefficient and the probability of connection. However, networked city infrastructures, e.g. water distribution networks (WDNs), are constrained by the spatial characteristics of the environment where they are laid. Therefore, networked infrastructures are classified as spatial networks and the classification of their connectivity structure requires a modification of the classic framework. To this purpose, the paper proposes a classification of WDNs using the neighbourhood nodal degree instead of the classic degree, the network size instead of the probability of connection and the classic average path length. The research will show that the clustering coefficient is not useful to describe the behavior of these constrained systems.
\end{abstract}

\section{Introduction}

The complex network theory (CNT) is useful to analyze the behavior of many systems in real world: relationships of individuals, Internet with hyperlinks, city infrastructures (e.g. streets, water systems), etc. Several network models have been proposed and developed in order to simplify the study of real systems, whose connectivity structures are defined using the nodal degree, the average path length, the clustering coefficient and the probability of connection.

\footnotetext{
${ }^{*}$ Corresponding author: antonietta.simone@poliba.it
} 
Erdos and Rényi (Erdös \& Rényi, 1959; Erdös \& Rényi, 1960) first studied the degree distribution of real systems introducing the random networks. In random networks, the degree is randomly distributed around an average value meaning that many nodes have a similar number of connections, i.e. the network is characterized by a high homogeneity. Random networks describe network features in a more realistic way with respect to regular networks, i.e. networks having a regular topology. The degree distribution of regular networks, in fact, is characterized by an absolute homogeneity, i.e. all nodes have the same degree. Later, Watts and Strogatz (Watts \& Strogatz, 1998) defined the small world networks based on Milgram's experiment (Milgram, 1967) on six degrees of separation of social networks, that is, to connect two nodes within a network requires at most six steps. They demonstrated the existence of the small world effect for the most part of real systems (WWW, social networks, etc.) starting from regular networks and replacing some of the links with others between different nodes, giving randomness to the network. Therefore, the small world networks present a certain level of homogeneity, which is lower than regular and higher than random networks. The degree distribution of small world networks is very similar to random networks; thus, a Poisson distribution of nodal degrees is assumed to cover from regular to random networks (Figure 1). In fact, for random networks, the Poisson distribution model shows that each pair of nodes is connected randomly with a probability $\mathrm{p}$, which generates a network having a great number of nodes with similar degrees. For regular networks, the probability $\mathrm{p}$ is zero and tends to increase with increasing random connections in the network; for small world networks, the probability is greater than the null value of regular networks but rather lower than values of random networks.
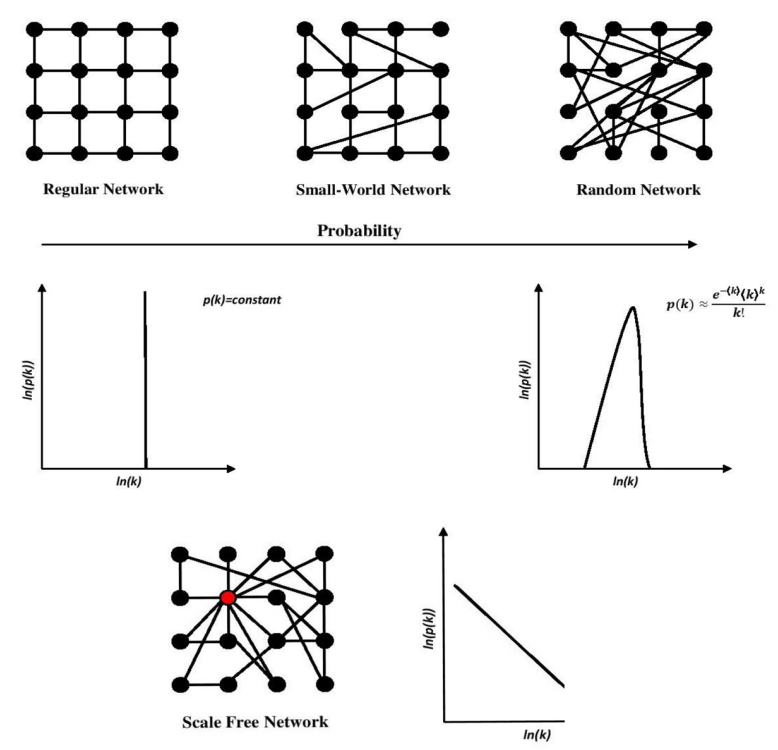

Figure 1. Degree distribution for regular, small world, random (bottom) and scalefree (down) networks

In the same period, Barabási and Albert (Barabási \& Albert, 1999; Albert, et al., 2000) introduced the scale-free networks, i.e. real networks with many nodes having a much lower degree than few nodes, named hubs. The degree distribution of scale-free networks is non-homogeneous, showing a Pareto distribution of nodal degrees, therefore they cannot be classified as random, small world or regular networks (Figure 1). 
Figure 1 shows that regular networks are highly ordered. This means that their shortest path length linking two nodes are very high, differently from what happens to real networks. Instead, the shortest path length for random network, which are highly random, are very low.

A real networked system, generally, has an average path length typical of small world networks, i.e. lower than regular network and greater than random networks. It is worth to note that also scale free networks can have a low average path length, similarly to small world ones, because the presence of hubs reduces the degree of separation among nodes, given that, generally, WDNs are not scale free networks (Giustolisi, et al., 2017).

The article shows the usefulness of the neighbourhood nodal degree instead of the classic degree (that is uninformative due to the very limited range of degree values), the network size instead of the probability of connection and the classic average path length in the classification of WDNs, highlighting the uselessness of the clustering coefficient.

\section{Classic Network Classification}

The degree distribution $P(k)$ is defined as the fraction of nodes in the network having degree $k$ :

$$
P(k)=\frac{n_{k}}{n}
$$

where $n_{k}$ is the number of nodes having degree $\mathrm{k}$ and $\mathrm{n}$ is the total number of nodes. The formulation of the Poisson model for degree distribution (Watts \& Strogatz, 1998) is

$$
P(k)=\left(\begin{array}{c}
n-1 \\
p
\end{array}\right) p^{k}(1-p)^{n-1-\langle k\rangle} \approx \frac{e^{-\langle k\rangle}\langle k\rangle^{k}}{k !}
$$

where $p$ is the probability of connection of nodes and $\langle k>$ is the average degree of the network. The formulation of the Pareto model for degree distribution is:

$$
P(k) \approx k^{-\gamma}
$$

where $\gamma$ is a constant generally ranging from 1.5 to 3 (Barthélemy, 2011 ).

Random and small world networks, modelled by the Poisson distribution, can be characterized by the clustering coefficient $C_{p}$ and the average path length $L_{p}$, normalized by clustering coefficient $C_{0}$ and average path length $L_{0}$ of the ring lattice regular network, respectively. Figure 2 shows that regular networks (with null p) have high $L_{p} / L_{0}$ and $C_{p} / C_{0}$, small world networks (with intermediate p) have a low $L_{p} / L_{0}$ and a high $C_{p} / C_{0}$ and random networks (with $p$ close to the unit value) have low both $L_{p} / L_{0}$ and $C_{p} / C_{0}$. Generally, the probability $p$ determines the average degree of the network,

$$
\langle k\rangle=p(n-1)
$$

The situation changes for WDNs whose average degree is determined by spatial constraints. It is possible to state that the average degree for WDNs ranges between 2 and 3, hence, it is possible to write,

$$
p=\frac{\langle k\rangle \approx 2.5}{n-1} \Rightarrow p \propto n^{-1}
$$


where the probability of connection is very low for the network connectivity structure of WDNs because of spatial constraints, besides being inversely proportional to the size of the network.
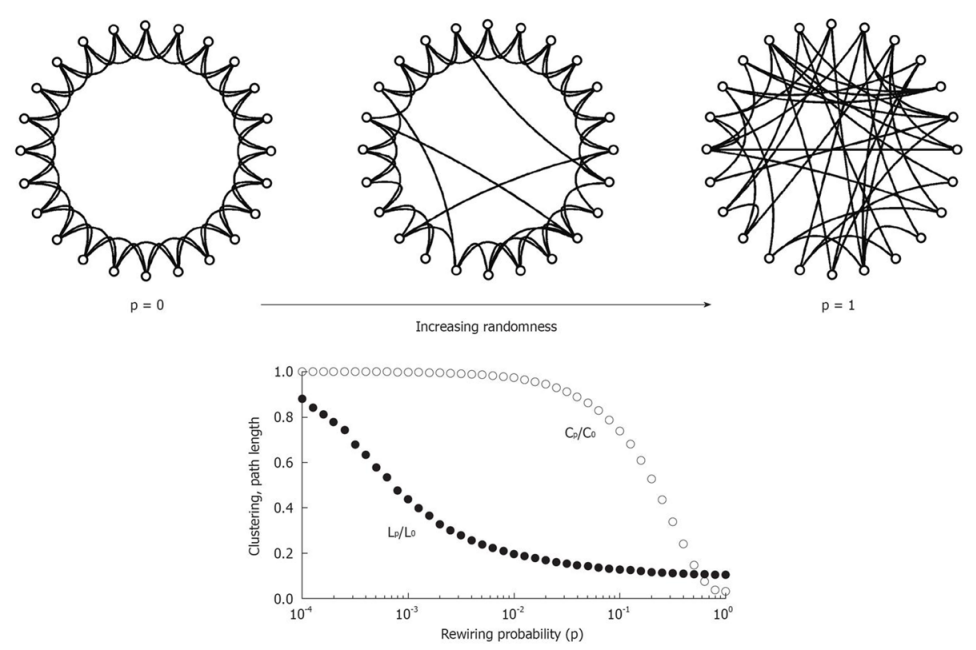

Figure 2. The explanation of small world networks with respect to regular and random networks.

\section{Characterization of network connectivity structures of WDNs}

WDNs are infrastructure networks whose evolution is constrained by urban limits. For these networks the topological information about the graph (stored in the adjacency matrix) and the spatial information about nodes are necessary. Spatial information relates to the construction process influenced by spatial constraints. In fact, spatial networks are generally manmade, and they progressively grow, filling the space, based on connection costs, nodal distances and constrains related to the impracticality of some connections (Buhl, et al., 2004). The main function of such networks is to allow the efficient circulation along edges and communication among nodes within the network. They need to be reliable with respect to random failures and intentional threats, and, therefore, they cannot present characteristics of scale free networks (Barthélemy, 2003). For the specific case of spatial networks related to city infrastructures, such as WDNs, the spatial constraints are buildings and streets, therefore the possible number of nodal degrees results strongly constrained as well as the maximum nodal degree. Consequently, the degree distribution can appear regular in some portion of the network based on an elementary topology, generally squared, corresponding to regular configuration of streets where pipes are installed. This fact further hampers the effectiveness in identifying the probability distribution for a network having a low maximum degree, which is generally lower than degree equal to ten.

Due to the very limited range of nodal degree values (i.e., between 3 and 7), the classification of such networks using the classic strategy based on the degree distribution is uninformative. In fact, such limited range of degree values does not allow the reliable identification of the relevant probability distribution function (i.e., Pareto or Poisson). To overcome these limitations and propose a good classification of these systems, the neighbourhood nodal degree (Giustolisi, et al., 2017) is used herein. It spans over a range of values greater than the standard nodal degree, resulting into a statistically more 
reliable classification of WDNs, exploiting the topological information of the nearest neighbours and allowing to reliably inferring probability distribution function. Furthermore, the characterization of network connectivity structures of WDNs considers the network size and the classic average path length for each network.

\subsection{Neighbourhood nodal degree}

Giustolisi et al. (Giustolisi, et al., 2017) proposed the neighbourhood nodal degree with the purpose of using the degree distribution concept but also overcoming its limits for infrastructures networks. The neighbourhood degree distribution of each node is the sum of the standard degrees of the topologically nearest (i.e., adjacent) nodes. The formulation of the neighbourhood degree is

$$
k_{n}(i)=\sum_{j \in N(i)} A_{i j} \cdot k(j)
$$

where $k_{n}(i)$ is the "neighbourhood" degree (involving adjacent nodes) of the i-th node, $A_{i j}$ are the elements of the adjacency matrix, $k(j)$ is the standard degree of the $j$-th node, and $N(i)$ is the topological neighbourhood of $i$-th node, i.e. the set of adjacent nodes.

The difference between the standard degree and the neighbourhood degree is that the first one measures the nodal connectivity with adjacent nodes in terms of number of links while the second one measures the nodal connectivity at the levels of neighbours. Considering that WDNs are infrastructure networks for which, for example, the behavior of single nodes is not relevant from a technical standpoint, this extension is helpful to guarantee a reliable analysis of these networks.

\section{Case studies}

Here 21 real WDNs are investigated, each following a Poisson distribution of neighbourhood nodal degree, as shown in Giustolisi et al. (Giustolisi, et al., 2017). Table 1 shows the Average path length $\left(L_{n}\right)$, the normalized one to ring lattice $\left(L_{n} / L_{0}\right)$, the Clustering coefficient $\left(C_{n}\right)$, the normalized one to ring lattice $\left(C_{n} / C_{0}\right)$ and the Meshdness coefficient $(M)$ for each network structure of the twenty-one WDNs. Networks are ordered according to their size.

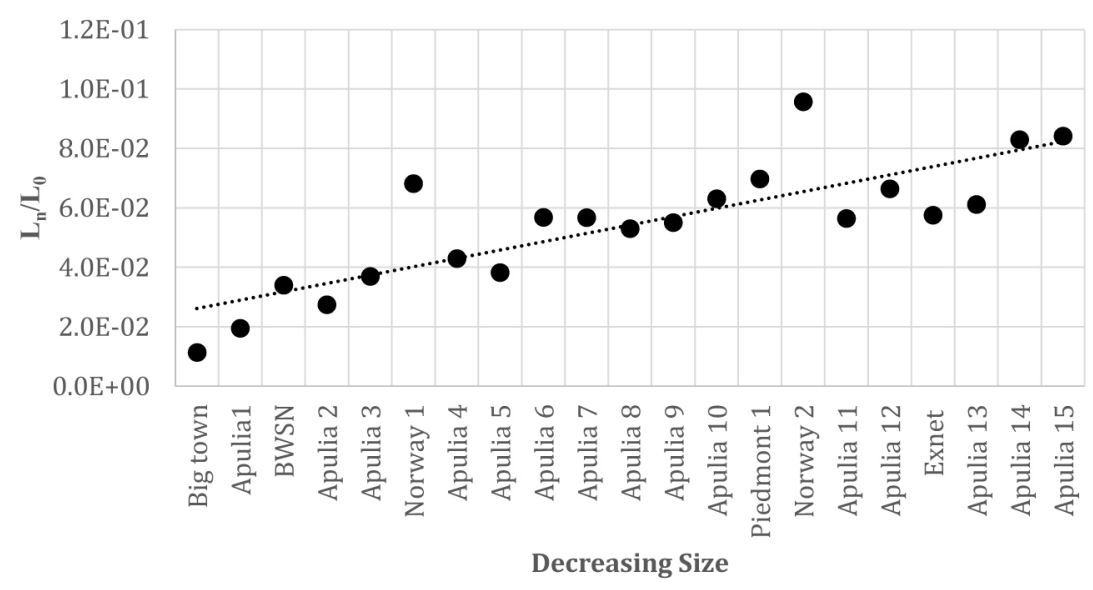

Figure 3. Normalized average path length $L_{n} / L_{0}$ versus decreasing network size for 21 real WDNs 
Table 1. Characterization of network connectivity structures of 21 real WDNs considering network size, average path length (normalized and not), clustering coefficient (normalized and not) and meshdness coefficient for each network.

\begin{tabular}{lllllll}
\hline WDN name & $\begin{array}{l}\text { Node } \\
\#\end{array}$ & {$\left[\boldsymbol{L}_{\boldsymbol{n}}\right]$} & {$\left[\boldsymbol{L}_{\boldsymbol{n}} \boldsymbol{L}_{\boldsymbol{n}}\right]$} & {$\left[\boldsymbol{C}_{\boldsymbol{n}}\right]$} & {$\left[\boldsymbol{C}_{\boldsymbol{n}} / \boldsymbol{C}_{\boldsymbol{o}}\right]$} & {$[\boldsymbol{M}]$} \\
\hline Big Town & 26761 & 63.36 & $1.14 \mathrm{E}-02$ & $3.30 \mathrm{E}-03$ & $1.54 \mathrm{E}-02$ & $9.97 \mathrm{E}-02$ \\
Apulia 1 & 18718 & 85.47 & $1.95 \mathrm{E}-02$ & $6.68 \mathrm{E}-04$ & $7.45 \mathrm{E}-03$ & $3.40 \mathrm{E}-02$ \\
BWSN & 12518 & 93.10 & $3.40 \mathrm{E}-02$ & $1.55 \mathrm{E}-02$ & $9.26 \mathrm{E}-02$ & $7.18 \mathrm{E}-02$ \\
Apulia 2 & 5288 & 31.38 & $2.75 \mathrm{E}-02$ & $2.71 \mathrm{E}-03$ & $1.52 \mathrm{E}-02$ & $7.84 \mathrm{E}-02$ \\
Apulia 3 & 5036 & 40.10 & $3.70 \mathrm{E}-02$ & $6.95 \mathrm{E}-04$ & $3.80 \mathrm{E}-03$ & $8.08 \mathrm{E}-02$ \\
Norway 1 & 5035 & 81.75 & $6.83 \mathrm{E}-02$ & $3.64 \mathrm{E}-04$ & $5.24 \mathrm{E}-03$ & $2.56 \mathrm{E}-02$ \\
Apulia 4 & 4242 & 39.13 & $4.30 \mathrm{E}-02$ & $1.38 \mathrm{E}-03$ & $7.45 \mathrm{E}-03$ & $8.24 \mathrm{E}-02$ \\
Apulia 5 & 4188 & 35.48 & $3.82 \mathrm{E}-02$ & $9.95 \mathrm{E}-04$ & $6.48 \mathrm{E}-03$ & $6.45 \mathrm{E}-02$ \\
Apulia 6 & 3547 & 46.07 & $5.69 \mathrm{E}-02$ & $0.00 \mathrm{E}+00$ & $0.00 \mathrm{E}+00$ & $4.73 \mathrm{E}-02$ \\
Apulia 7 & 3000 & 40.05 & $5.68 \mathrm{E}-02$ & $8.33 \mathrm{E}-04$ & $9.93 \mathrm{E}-03$ & $3.17 \mathrm{E}-02$ \\
Apulia 8 & 2968 & 34.38 & $5.31 \mathrm{E}-02$ & $3.37 \mathrm{E}-04$ & $1.99 \mathrm{E}-03$ & $7.30 \mathrm{E}-02$ \\
Apulia 9 & 2895 & 34.65 & $5.51 \mathrm{E}-02$ & $0.00 \mathrm{E}+00$ & $0.00 \mathrm{E}+00$ & $7.59 \mathrm{E}-02$ \\
Apulia 10 & 2810 & 37.69 & $6.31 \mathrm{E}-02$ & $2.55 \mathrm{E}-03$ & $1.31 \mathrm{E}-02$ & $8.87 \mathrm{E}-02$ \\
Piedmont 1 & 2784 & 46.71 & $6.98 \mathrm{E}-02$ & $5.99 \mathrm{E}-04$ & $1.09 \mathrm{E}-02$ & $2.00 \mathrm{E}-02$ \\
Norway 2 & 2520 & 57.38 & $9.58 \mathrm{E}-02$ & $6.96 \mathrm{E}-03$ & $9.92 \mathrm{E}-02$ & $2.62 \mathrm{E}-02$ \\
Apulia 11 & 2403 & 28.91 & $5.65 \mathrm{E}-02$ & $1.39 \mathrm{E}-03$ & $7.18 \mathrm{E}-03$ & $8.71 \mathrm{E}-02$ \\
Apulia 12 & 1918 & 28.40 & $6.65 \mathrm{E}-02$ & $8.69 \mathrm{E}-04$ & $5.89 \mathrm{E}-03$ & $6.16 \mathrm{E}-02$ \\
Exnet & 1776 & 19.76 & $5.76 \mathrm{E}-02$ & $4.38 \mathrm{E}-02$ & $1.58 \mathrm{E}-01$ & $1.48 \mathrm{E}-01$ \\
Apulia 13 & 1762 & 22.65 & $6.12 \mathrm{E}-02$ & $1.23 \mathrm{E}-03$ & $5.94 \mathrm{E}-03$ & $9.58 \mathrm{E}-02$ \\
Apulia 14 & 1263 & 23.18 & $8.30 \mathrm{E}-02$ & $1.06 \mathrm{E}-03$ & $6.79 \mathrm{E}-03$ & $6.58 \mathrm{E}-02$ \\
Apulia 15 & 1263 & 23.88 & $8.42 \mathrm{E}-02$ & $0.00 \mathrm{E}+00$ & $0.00 \mathrm{E}+00$ & $5.71 \mathrm{E}-02$
\end{tabular}

The diagram in Figure 3 clearly demonstrates that $L_{n} / L O$ increases with decreasing of the network size as also shown by the trend line there reported, meaning that decreasing the WDN size, the network structure exhibits a higher regularity. This aspect shows that the probability $p$ is not an effective parameter for our systems because naturally decreasing with the network size $n$. Therefore, $n$ has substituted $p$ in Watts-Strogatz (Watts \& Strogatz, 1998) diagram of Figure 2.

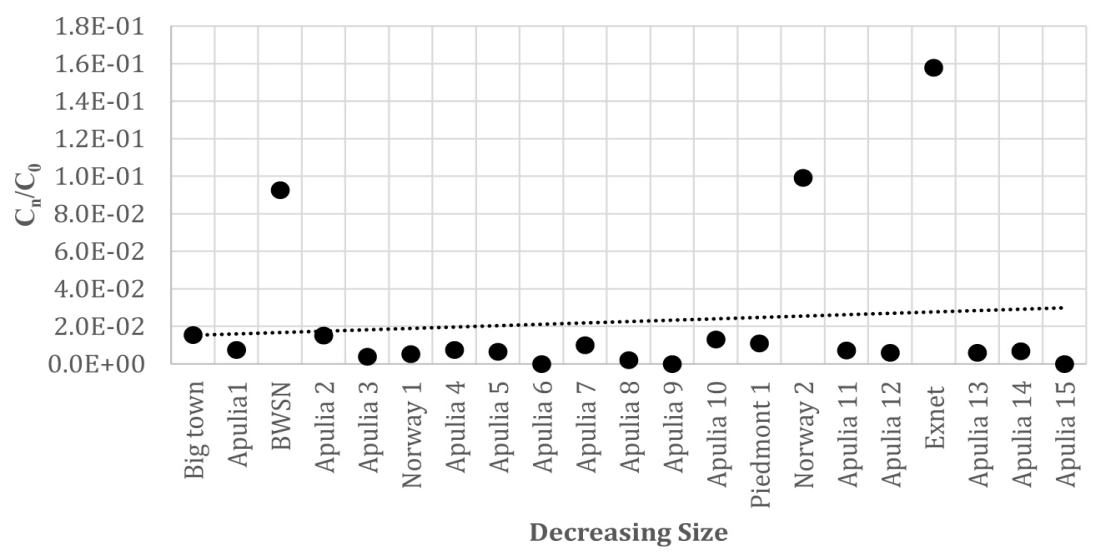

Figure 4. Normalized clustering coefficient $C_{n} / C_{0}$ versus decreasing network size for 21 real WDNs in (Giustolisi, et al., 2017). 


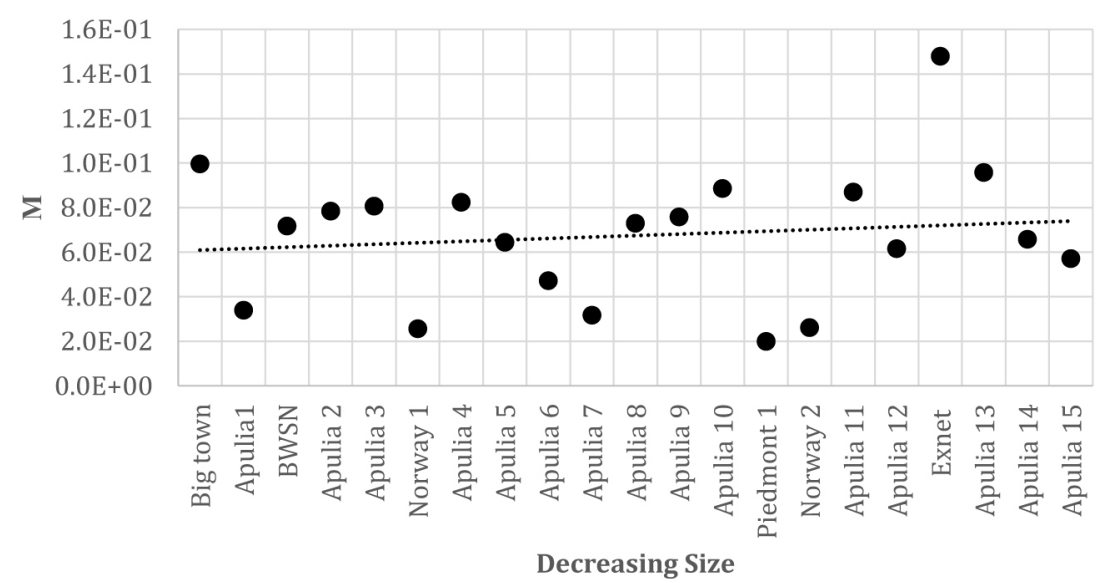

Figure 5. Meshdness coefficient versus decreasing network size for 21 real WDNs in (Giustolisi, et al., 2017).

The normalized clustering coefficient $C_{n} / C_{0}$ versus decreasing network size in Figure 4 shows, instead, that the parameter is very low and not depending on network size. This because $C_{n}$ is based on cliques of size three (i.e. triangles) and the spatial constraints of infrastructure networks limit the number of elementary topologies. For example, $C_{n}$ is null for Apulia 6, Apulia 9 and Apulia 15 networks as also reported in Table 1. The meshdness coefficient $M$ versus decreasing network size (Figure 5) also shows that parameters are very low and not depending on network size (see the tendency lines).

\section{Conclusions}

The paper proposes a different framework to describe the WDNs connectivity structure, also considering their spatial constraints, returning important results in terms of classification and structural properties of WDNs.

The neighbourhood nodal degree replaces the classic degree to guarantees a more robust identification of a specific statistical distribution (an evident Poisson distribution for the 21 analysed WDNs (Giustolisi, et al., 2017)). Furthermore, the probability of connection $p$ is replaced by the network size $n$ due to the low value of the average nodal degree $(\approx 2.5)$. Results show that the average path length, i.e. the regularity of a network structure, increases with decreasing the network size. This aspect is of considerable importance in the analysis of temporal networks, i.e. networks that vary their structure (increase in nodes and edges) over time. For WDNs this happens in the normal evolution of the urban centre over decades and indicates that original networks (few nodes and pipes) have a higher average path length value, i.e. a greater regularity, with respect to the same network evolved (Giustolisi, et al., 2017; Barthélemy, 2011 ).

These results can be also useful for analysing WDNs emerging behaviour, for example related to their vulnerability to random failures and intentional threats. The fact that most of the studied WDNs can be modelled using the Poisson distribution of the neighbourhood nodal degree means that they present a significant structural resistance to random failures and intentional threats as connectivity structure. Different approaches can be used to analyse the WDN behaviour with respect to vulnerability, but the classic classification of the connectivity structure nodal degree-based has wide implications for classifying them as small world ore purely random considering temporal evolution or for assessing 
vulnerability using some technical weights (e.g. hydraulic flows) while analysing their connectivity structure.

\section{Reference}

Albert, R., Jeong, H. \& Barabasi, A.-L., 2000. Error and attack tolerance of complex networks. Nature, Issue 406, pp. 378-382.

Barabási , A. \& Albert, R., 1999. Emergence of scaling in random networks. Science, Issue 286, p. 509-11.

Barthélemy, M., 2003. Crossover from Scale-Free to Spatial Networks,. Europhys. Lett., 63(6), pp. 915-921.

Barthélemy, M., 2011 . Spatial networks. Physics Reports, 499(1-3), pp. 1-101.

Buhl, J. et al., 2004. Efficiency and robustness in ant networks of galleries. Eur. Phys. J.B., 42(1), pp. 123-129.

Erdös, P. \& Rényi, 1960. On the evolution of random graphs. Publ. Math. Inst. Hung. Ac. Sci. 5, pp. 17-60.

Erdös, P. \& Rényi, A., 1959. On random graphs. Publ. Math., pp. 290-297.

Giustolisi, O., Simone, A. \& Ridolfi, L., 2017. Network structure classification and features of water distribution systems. Water Resources Research, Issue 53, p. 3407-3423.

Milgram, S., 1967. The Small World Problem. Psychology today, p. 60-67.

Watts, D. \& Strogatz, D., 1998. Collective dynamics of small-world networks. Nature, p. 393-440. 